\title{
Plasma cytokines, chemokines and cellular immune responses in pre-school Nigerian children infected with Plasmodium falciparum
}

Cariosa Noone ${ }^{1}$, Michael Parkinson ${ }^{1}$, David J Dowling ${ }^{1}$, Allison Aldridge ${ }^{1}$, Patrick Kirwan² ${ }^{2}$, Síle F Molloy², Samuel O Asaolu ${ }^{3}$, Celia Holland ${ }^{2}$ and Sandra M O'Neill ${ }^{1 *}$

\begin{abstract}
Background: Malaria is a major cause of morbidity and mortality worldwide with over one million deaths annually, particularly in children under five years. This study was the first to examine plasma cytokines, chemokines and cellular immune responses in pre-school Nigerian children infected with Plasmodium falciparum from four semi-urban villages near lle-lfe, Osun State, Nigeria.

Methods: Blood was obtained from 231 children (aged 39-73 months) who were classified according to mean P. falciparum density per $\mu$ l of blood (uninfected $(n=89)$ ), low density $(<1,000, n=51)$, medium density $(1,000-10,000, n=65)$ and high density $(>10,000, n=22))$. IL-12p70, IL-10, Nitric oxide, IFN- $\gamma$, TNF, IL-17, IL-4 and TGF- $\beta$, C-C chemokine RANTES, MMP-8 and TIMP-1 were measured in plasma. Peripheral blood mononuclear cells were obtained and examined markers of innate immune cells (CD14, CD36, CD56, CD54, CD11c AND HLA-DR). T-cell sub-populations (CD4, CD3 and $\gamma \delta T C R)$ were intracellularly stained for IL-10, IFN- $\gamma$ and TNF following polyclonal stimulation or stimulated with malaria parasites. Ascaris lumbricoides was endemic in these villages and all data were analysed taking into account the potential impact of bystander helminth infection. All data were analysed using SPSS 15 for windows and in all tests, $p<0.05$ was deemed significant.

Results: The level of $P$. falciparum parasitaemia was positively associated with plasma IL-10 and negatively associated with IL-12p70. The percentage of monocytes was significantly decreased in malaria-infected individuals while malaria parasitaemia was positively associated with increasing percentages of $\mathrm{CD} 54^{+}, \mathrm{CD} 11 \mathrm{c}^{+}$and $\mathrm{CD}_{56}{ }^{+}$cell populations. No association was observed in cytokine expression in mitogen-activated T-cell populations between groups and no malaria specific immune responses were detected. Although A. lumbricoides is endemic in these villages, an analysis of the data showed no impact of this helminth infection on $P$. falciparum parasitaemia or on immune responses associated with P. falciparum infection.

Conclusions: These findings indicate that Nigerian children infected with $P$. falciparum exhibit immune responses associated with active malaria infection and these responses were positively associated with increased $P$. falciparum parasitaemia.
\end{abstract}

Keywords: Cytokines, Chemokines, Cellular responses, Plasmodium falciparum, Children, Nigeria, Ascaris lumbricoides

\footnotetext{
* Correspondence: sandra.oneill@dcu.ie

'Parasite Immune Modulation Group, School of Nursing and Human

Sciences, Faculty of Science and Health, Dublin City University, Glasnevin

Dublin 9, Ireland

Full list of author information is available at the end of the article
} 


\section{Background}

Plasmodium falciparum malaria accounts for approximately 250-300 billion clinical cases of malaria worldwide and is highly endemic in Africa [1]. Approximately one in every five child deaths in Africa are due to malaria with the risk of cerebral malaria being highest in children aged two to four years. Natural acquired immunity rarely occurs before two years and its development is associated with increasing age, which correlates with a reduction in mortality rates due to the more severe forms of $P$. falciparum infection [2]. It is, therefore, important that immune responses in young children are examined in order to further define immunological association with $P$. falciparum infection.

Malaria infection is predominantly characterized by a $\mathrm{T}$ helper 1 (Th1) response and the production of proinflammatory cytokines such as IL-12-p70, interferon gamma (IFN- $\gamma$ ) and tumour necrosis factor (TNF). These inflammatory cytokines are considered critical in controlling parasitaemia, especially during the early stages of $P$. falciparum infection $[3,4]$. Conversely during chronic malaria infection, if these robust inflammatory responses are not tightly regulated, they can lead to immunopathology and severe forms of malaria $[5,6]$. Regulatory cytokines, including interleukin (IL)-10 and transforming growth factor beta (TGF- $\beta$ ) were shown to be important in dampening down $\mathrm{T}$ helper (Th) 1 inflammatory responses associated with immune pathology in the more severe forms of $P$. falciparum infection $[5,6]$. There also a range of other mediators, such as IL-17, IL-4, nitric oxide, C-C chemokine RANTES, matrix metalloproteinases 8 (MMP8s) and tissue inhibitor of metalloproteinases 1 (TIMP1) that have been linked to disease severity in malaria-infected individuals [7-9].

Malaria infection is strongly influenced by the release of inflammatory mediators from innate immune cells where early interactions between blood-stage parasites and these are critical in controlling parasitaemia and the subsequent elimination of infection $[5,6]$. Innate immune cells including antigen presenting cells, such as dendritic cells, and macrophages are an early source of proinflammatory cytokines, such as IL-12 and TNF. Other innate immune cells such as natural killer cells and $\gamma \delta$-T-cells are an early source of IFN- $\gamma$. These cells, through the release of inflammatory mediators and from cell-to-cell contact with naïve T-cells, also shape the adaptive immune response.

This is the first study to assess immune responses during uncomplicated malaria infection in Nigerian pre-school children in four semi-urban villages near IleIfe, Osun State, Nigeria [10]. Plasmodium falciparum infection is endemic in this region with a high prevalence in pre-school children [11]. Recent studies have also shown a $25 \%$ prevalence of Ascaris lumbricoides in this cohort [12]. Since helminth infection can impact upon the outcome of malaria infection [13-15] the potential impact of $A$. lumbricoides upon P. faliciparum parasitaemia and its associated immune responses were examined.
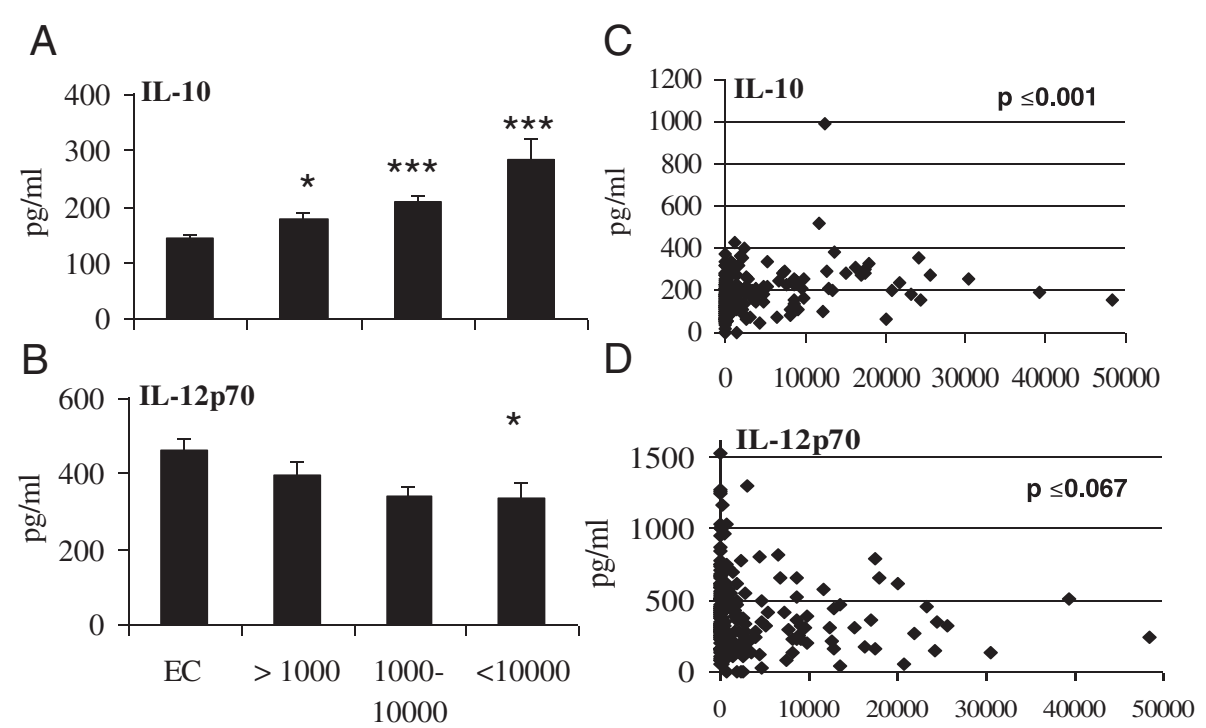

Figure 1 Plasmodium falciparum parasitaemia was positively associated with IL-10 and negatively associated with IL-12p70 levels in the plasma of infected children. Mean plasma levels of (A) IL-10 and (B) IL-12p70 were determined by ELISA for each group (low ( $<1,000$; $n=51)$, medium (1,000-10,000; $n=65)$ and high ( $>10,000 ; n=22)$ mean parasite density (per $\mu$ l of blood) and compared to endemic controls (EC) $(\mathrm{n}=89) .{ }^{*}, p \leq 0.05 ;{ }^{* *}, p \leq 0.01$ and ${ }^{* * *}, p \leq 0.001$ (ANOVA). Parasitaemia was plotted against (C) IL-10 and (D) IL-12p70 and association between variables was assessed using regression analysis. $p<0.05$ was deemed significant. 


\section{Methods}

Study design and participants

231 blood samples were obtained from children at the final time point in a double-blind placebo-controlled randomized trial on children aged 39-73 months in four semi-urban villages, Akinlalu, Ipetumodu, Moro and Edunabon, near Ile-Ife, Osun State, Nigeria. Details of the study area, design and participants were published previously [10]. This current sub-study was to examine immune responses associated with $P$. falciparum infection and examine the impact of $A$. lumbricoides. Data were available on children's age and infection status for $P$. falciparum and $A$. lumbricoides infection. Children who suffered from severe malaria were treated and excluded from the study and therefore only individuals with uncomplicated malaria were included (malaria parasitaemia and fever $>37.5^{\circ} \mathrm{C}$ ) [10]. The study protocol was approved by the Ethics and Research Committee, Obafemi Awolowo University Teaching Hospitals' Complex, Ile-Ife, Nigeria.

\section{Isolation of peripheral blood mononuclear cells}

Ten $\mathrm{ml}$ of blood (in tubes containing heparin) was obtained from 231 children, ranging in age from 39-73 months. Peripheral blood mononuclear cells (PBMCs) and plasma were obtained following histopaque (SigmaAldrich, St Louis, MO, USA) density gradient centrifugation. PBMCs were collected and stored in liquid nitrogen and plasma samples were stored at $-80^{\circ} \mathrm{C}$.
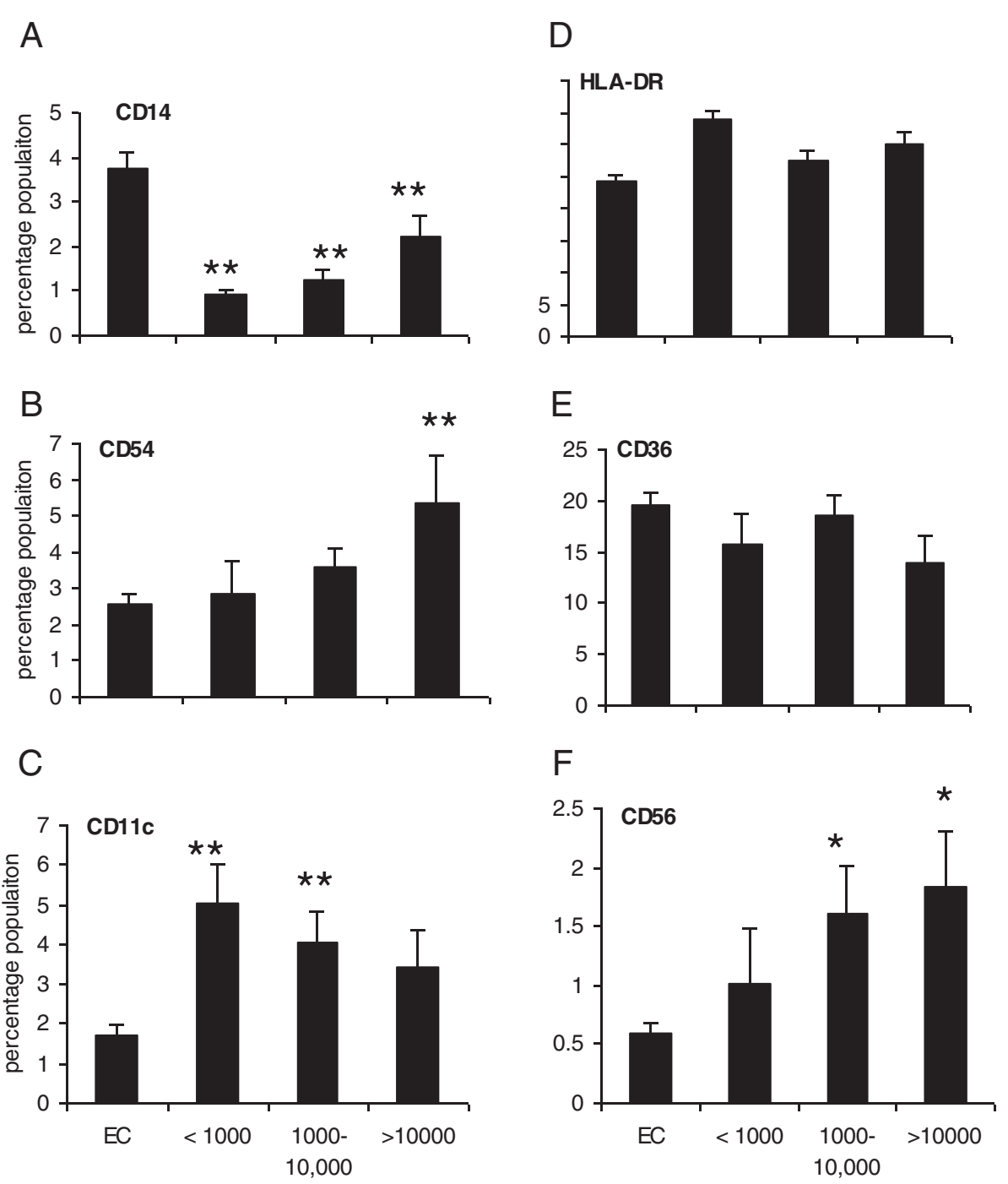

Figure 2 Malaria infection was associated with decreased $C D 14^{+}$percentages and enhanced percentages of CD11 ${ }^{+}, C D 54^{+}, C D 56$ but not HLA-DR ${ }^{+}$and $\mathrm{CD} 6^{+}$cell populations. PBMCs were stained extracellularly for CD14 (A), CD54 (B) CD11C (C) HLA-DR (D), CD36 (E) and CD56 (F) cell expression for each group (low $(<1,000 ; n=51)$, medium $(1,000-10,000 ; n=65)$ and high $(>10,000 ; n=22)$ mean parasite density (per $\mu$ l of blood)) was determined by flow cytometry and compared to endemic controls (EC). ${ }^{* *} p \leq 0.01$ (ANOVA). 


\section{ELISA}

Human IFN- $\gamma$, IL-10, TGF- $\beta$, TNF, IL-4 and IL-12p70 Opti-EIA kits (BD Biosciences) and human IL-17, RANTES, MMP-8 and TIMP-1 DuoSet ELISA Developmental Kits (R\&D, Minneapolis, MN, USA) were used to quantify cytokine, chemokine and metalloproteinase levels in plasma samples as described per manufacturers instructions. NO levels were also measured in plasma using the Greiss Reagent System (Promega, Madison, WI, USA).

\section{Flow cytometry and in vitro culture}

The following mAbs were used for cells surface staining and intracellular cytokine staining: FITC-conjugated anti- CD4, CD14, CD36, CD56, IFN- $\gamma$; PE-conjugated anti- $\gamma \delta \mathrm{TCR}, \mathrm{CD} 54, \mathrm{IL}-10$; and APC-conjugated antiCD3, CD11c, HLA-DR, IL-2 and TNF (eBiosciences, San Diego, CA, USA). Isotype controls included FITCconjugated mouse IgG1, IgM, IgG2a, IgG2b; PEconjugated mouse IgG1, IgG2b and APC-conjugated mouse IgG1, IgG2b and rat IgG2a (eBiosciences). PBMCs were thawed and cultured in complete RPMI containing $10 \%$ FCS (foetal calf serum), 1\% L-glutamine and $1 \%$ penicillin/streptomycin solution (Bio-sciences Ltd, Co. Dublin, Ireland). For intracellular cytokine staining, PBMCs were stimulated with $50 \mathrm{ng} / \mathrm{ml}$ PMA (Phorbol 12-myristate 13-acetate) and $1 \mathrm{mg} / \mathrm{ml}$ ionomycin for $4 \mathrm{~h}$ and to block cytokine secretion, $10 \mathrm{mg} / \mathrm{ml}$ Brefeldin A (Sigma) was added to the culture media. Cells were then washed and stained with cell surface mAbs, fixed with 4\% PFA and permeabilized with $0.2 \%$ saponin (Sigma), before incubation with antibodies for IL-12, IFN- $\gamma$, IL-10 and TNF cytokines. Appropriately labelled isotype-matched antibodies were used as controls. Acquisition was performed using a FACSCalibur flow cytometer (BD Biosciences), and analysis of results performed using FlowJo software (Tree Star). A sample of gating strategy for $\mathrm{T}$ Cells in shown in Additional file 1.

PBMCs $\left(1 \times 10^{6}\right.$ cells $\left./ \mathrm{ml}\right)$ from a cohort of children were also cultured on a 24-well plate with mycoplasma free $P$. falciparum parasites (at a ratio of 1:5), which were extracted from cell culture by saponin lysis $(0.15 \%)$, were kindly provided by Dr Alison Creasey, University of Edinburgh, Scotland. After three days, cell culture supernatants were harvested and frozen for subsequent measurement of IFN- $\gamma$, IL-10, and IL- 5 by commercial ELISA.

\section{Statistical analysis}

All data were analysed using SPSS 15 for windows. Percentage data were normalized prior to analysis by Arcsin transformation. Skewed data were normalized prior to analysis by log transformation. For differences between multiple groups, one-way ANOVA with Post-hoc testing by Tukey's HSD test was used. For data with more than one factor, factorial ANOVA was used. Association between variables was assessed using regression analysis. For differences between two treatments 2-tailed Student $t$ test was used. In all tests, $p<0.05$ was deemed significant.

\section{Results}

Plasmodium falciparum parasitaemia was positively associated with IL-10 and negatively associated with IL12 p70 levels in the plasma of infected children Children infected with P. falciparum were divided into groups based upon the mean $P$. falciparum density per $\mu \mathrm{l}$ of blood (uninfected $(\mathrm{n}=89)$, low density $(<1,000, \mathrm{n}$ $=51$ ), medium density $(1,000-10,000, \mathrm{n}=65)$ and high density $(>10,000, \mathrm{n}=22)$ ) to determine if an association exists between parasitaemia and plasma immune factors

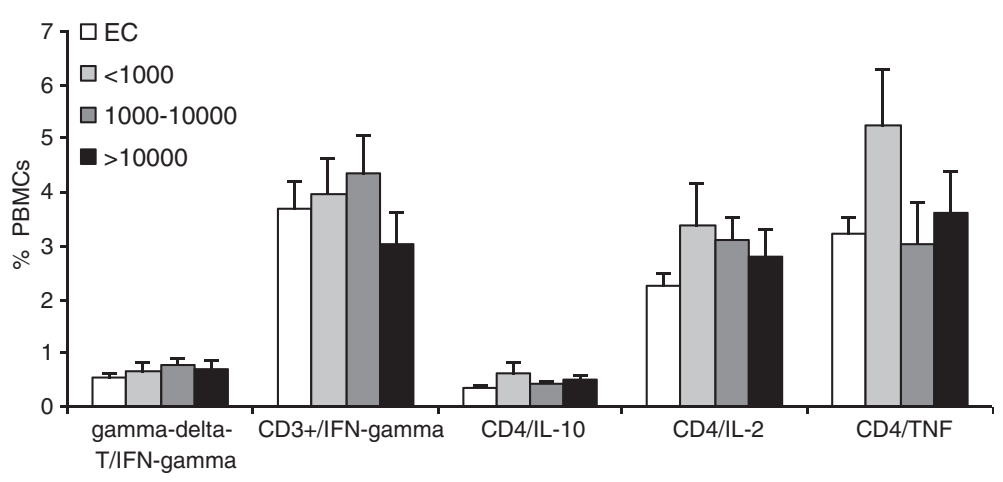

Figure 3 Malaria infection was not correlated with an increase in the secretion of IFN- $\gamma$, TNF, IL-10 and IL-2 in mitogen-activated T-cell populations. PBMCs were stimulated for $4 \mathrm{~h}$ with PMA and ionomycin. Cells were washed and stained for cell surface expression of $\gamma \delta T C R$, CD3 and CD4 and were intracellular stained for IFN- $\gamma, \mathrm{IL}-2, \mathrm{IL}-10$ or TNF. Appropriate isotype controls were included to define parameter gates. PBMCs were gated on the lymphocyte population and the percentage of positive cells for each group $($ low $(<1,000 ; n=51)$, medium $(1,000-10,000 ; n=$ 65) and high (>10,000; $n=22$ ) mean parasite density (per $\mu$ l of blood) were also compared to uninfected endemic controls (EC). 


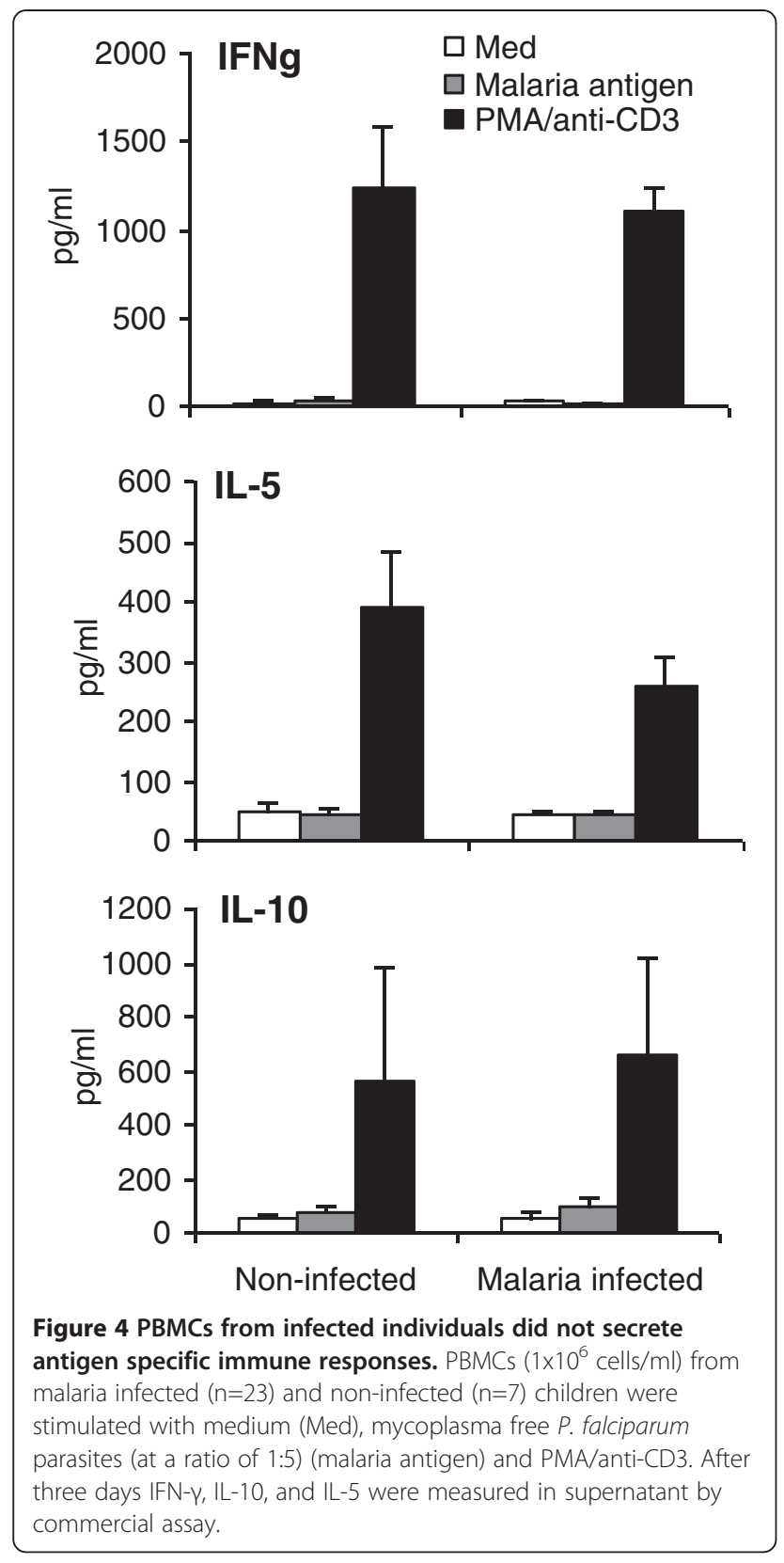

(Figure 1A-B, Additional file 2). A reciprocal relationship between IL-10 (low density ( $\mathrm{p} \leq 0.01)$, medium ( $\mathrm{p} \leq 0.001$ ) and high density ( $\mathrm{p} \leq 0.001$ ) (Figure $1 \mathrm{~A}$ ) and IL-12p70 levels (Figure 1B; high density $\mathrm{p} \leq 0.05$ ) was observed. Regression analysis revealed that increases in IL-10 were significantly associated with increased $P$. falciparum parasitaemia ( $P \leq 0.001$, Figure $1 C)$, however, IL-12p70 was not negatively associated with $P$. falciparum parasitaemia $(P \leq 0.067$, Figure 1D). Nitric oxide, IFN- $\gamma$, TNF IL-17, IL-4 and TGF- $\beta$, RANTES, MMP- 8 and TIMP-1 in plasma was not associated with $P$. falciparum parasitaemia (Additional file 2).

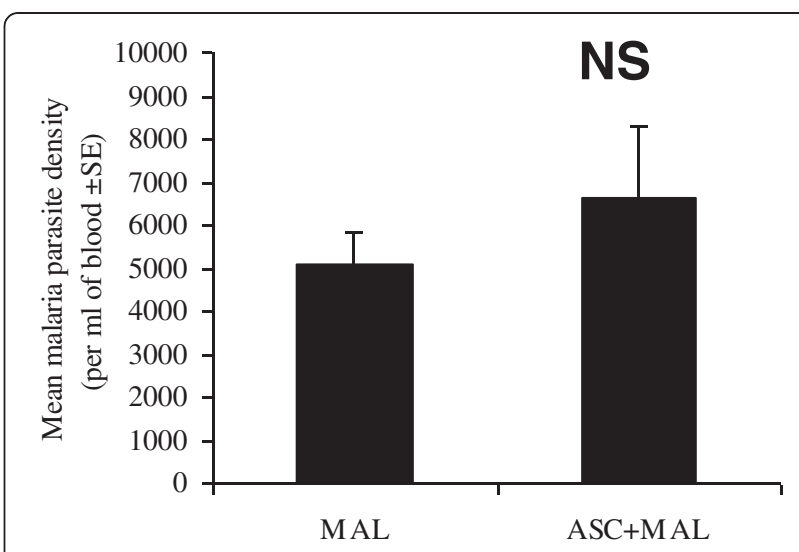

Figure 5 Ascaris lumbricoides infection did not impact upon Plasmodium falciparum parasitaemia. Mean P. falciparum density (per $\mu l$ of blood) was measured from malaria infected (MAL) and Ascaris and malaria co-infected (ASC+MAL) children by Giemsa stained blood slides ( $\mathrm{n}=109$ for MAL; $\mathrm{n}=32$ for $\mathrm{ASC}+\mathrm{MAL}$ ) NS = non-significant.

Malaria infection was associated with a decrease in the percentage of monocytes and enhanced percentages of $\mathrm{CD}_{11 \mathrm{c}^{+}}, \mathrm{CD}^{\circ} 4^{+}, \mathrm{CD}^{+} 6^{+}$but not $\mathrm{HLA}^{-D R^{+}}$and $\mathrm{CD} 6^{+}$cell populations

Previous studies have shown that $P$. falciparum infection is associated with changes in antigen presenting cell populations (APCs) [16]. Here, the percentage of $\mathrm{CD} 4^{+}$monocytes (precursor dendritic cells (DCs)/ macrophage) in PBMCs for each group were examined. All groups displayed significantly lower percentages of $\mathrm{CD} 4^{+}$cells $(<4 \%)$ compared to endemic controls (5-7\%) (Figure 2A; $P \leq 0.01$ for all infected groups). To dissect the APC subsets associated with malaria infection specific surface markers including HLA-DR (indicative of an active infection), CD11c (myeloid marker found on DCs), CD54 (also known as ICAM-1) (Intercellular Adhesion Molecule 1, is important for lymphocyte-APC binding and has been shown to be upregulated on APC during malaria infection) and CD36 (involved in phagocytosis of malaria infected red blood cells) were examined [17-21]. The percentage of $\mathrm{CD}_{54}{ }^{+}$populations were associated with high density $P$. falciparum parasitaemia only (Figure $2 \mathrm{~B} ; P \leq 0.01$ ) while the percentage of $\mathrm{CD}_{11 \mathrm{c}^{+}}$populations were associated with medium $(P \leq 0.01)$ and high $(P \leq 0.01)$ but not low density $P$. falciparum parasitaemia (Figure 2C). No association was observed between $\mathrm{HLA}-D R^{+}$(Figure 2D) and CD36 ${ }^{+}$cells (Figure 2E) and parasitaemia.

Since an increase in IFN- $\gamma$ was not detected in plasma cells that are known to produce IFN- $\gamma$ were examined to determine if there was a decrease in this population. Early IFN- $\gamma$ is important in the control of $P$. falciparum parasitaemia and studies have shown 

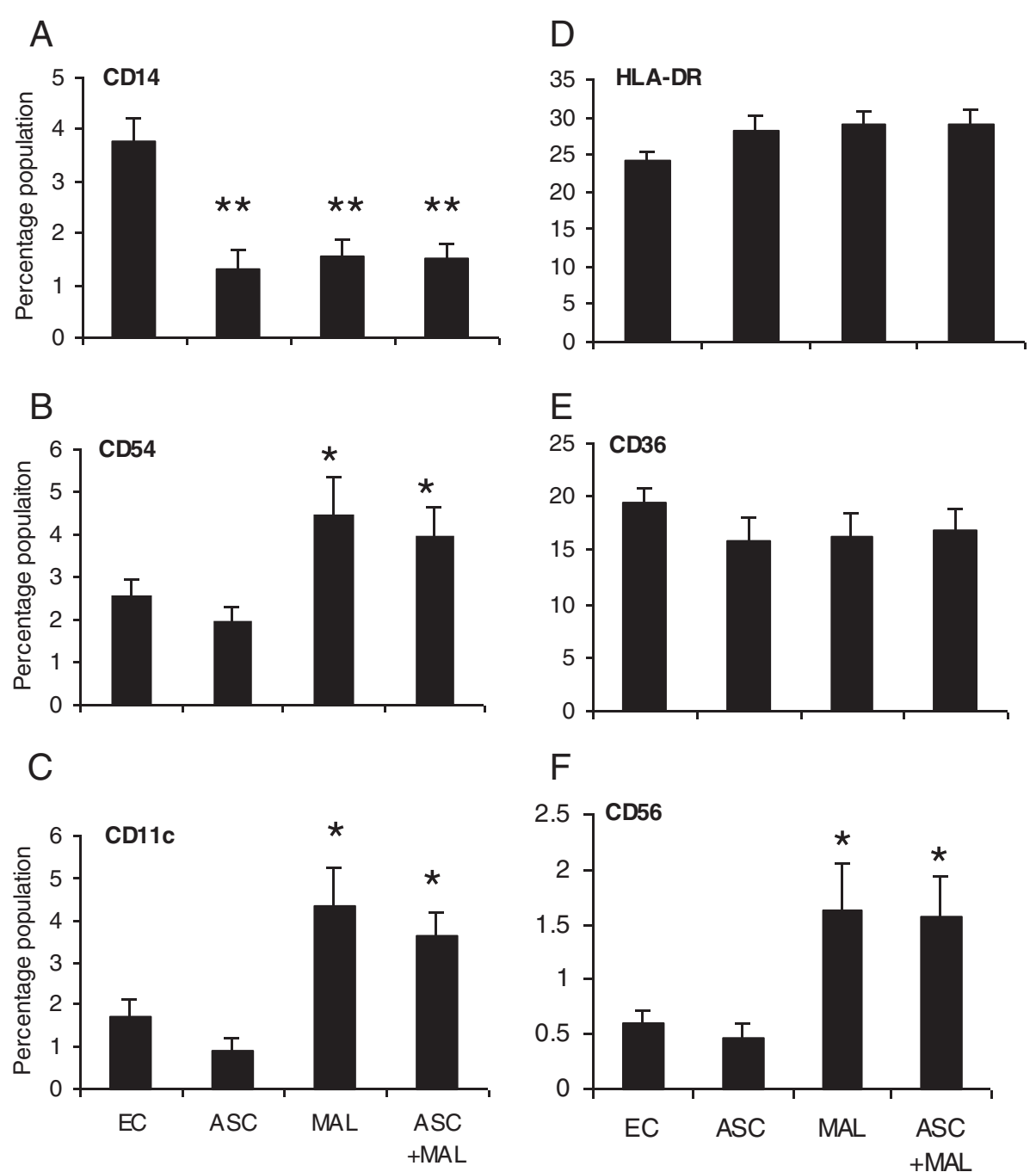

Figure 6 Ascaris lumbricoides infection did not alter the percentage of CD14 ${ }^{+}$CD11 ${ }^{+}$, CD54 $4^{+}, \mathrm{CD} 56 \mathrm{HLA}-\mathrm{DR}{ }^{+}$and CD36 ${ }^{+}$cell populations in-co-infected individuals. PBMCs were stained extracellularly for CD14 (A), CD54 (B) CD11C (C), HLA-DR (D), CD36 (E) and CD56 (F) cell expression for each group Ascaris infection only (ASC), Malaria infection only (MAL) and co-infected (ASC + MAL) was determined by flow cytometry and compared to endemic controls (EC). $p \leq 0.05 ;{ }^{* *} p \leq 0.01$ (ANOVA) and MAL group was compared to the ASC + MAL group (no significant differences).

that in the early stages of infection $\mathrm{CD}^{+} 6^{+}$natural killer cells (NK) and other leukocytes expressing the CD56 marker are good sources of IFN- $\gamma$ [22]. The percentage of $\mathrm{CD}^{+} 6^{+}$cells in PBMCs were measured and these cells were positively associated with $P$. falciparum parasitaemia with significant increases in cell percentages for medium $(P \leq 0.05)$ and high density $P$. falciparum parasitaemia $(P \leq 0.05)$ (Figure $2 \mathrm{~F})$.

Malaria infection was not associated with increased secretion of IFN- $\gamma$, TNF, IL-10 and IL-2 in mitogenactivated T-cell populations and no parasite specific immune responses were detected

PBMCs were polyclonally activated with PMA and ionomycin and intracellular cytokine staining for IFN- $\gamma$,
IL-10, IL-2 and TNF was performed. In order to evaluate the phenotype of the $\mathrm{T}$ cells present, cells were also stained for CD3, CD4, and $\gamma \delta$ T-cells. All groups expressed significantly high levels of IFN- $\gamma$ and TNF and low, but significant, levels of IL-10 and IL-2 following stimulation. There were no differences observed between the groups (Figure 3 ).

PBMCs $\left(1 \times 10^{6}\right.$ cells $\left./ \mathrm{ml}\right)$ from malaria infected $(\mathrm{n}=23)$ and non-infected $(\mathrm{n}=7)$ individuals were stimulated with mycoplasma free $P$. falciparum parasites (at a ratio of 1:5) and after three days IFN- $\gamma$, IL-10, and IL- 5 were measured in supernatant. No parasite specific immune responses were detected while cPBMCs were capable of secreting all cytokines tested in response to our positive control PMA/ anti-CD3 (Figure 4). 




Figure 7 Ascaris lumbricoides infection did not alter IFN- $\gamma$, TNF, IL-10 and IL-2 expression in mitogen-activated T-cell populations. PBMCs were stimulated for $4 \mathrm{~h}$ with PMA and ionomycin. Cells were washed and stained for cell surface expression of $\gamma \delta T C R, C D 3$ and CD4 and were intracellular stained for IFN-Y, IL-2, IL-10 or TNF-a. Appropriate isotype controls were included to define parameter gates. PBMCs were gated on the lymphocyte population and the percentage of positive cells for each group Ascaris infection only (ASC), Malaria infection only (MAL) and co-infected (ASC + MAL) was determined by flow cytometry and compared to uninfected endemic controls (EC) and MAL group was compared to the (ASC + MAL).

Low intensity Ascaris lumbricoides infection did not impact upon Plasmodium falciparum parasitaemia or its associated immune responses

The data was reanalysed taking into account Ascaris infection (uninfected $(\mathrm{n}=69)$, Ascaris only $(\mathrm{n}=21)$, malaria only $(\mathrm{n}=109)$, Ascaris and malaria $(\mathrm{n}=32))$. The prevalence of $A$. lumbricoides was $23 \%$ and all $A$. lumbricoides-infected children had a low intensity infection $(<3,700$ eggs per gram (epg)). Plasmodium falciparum percentages in the blood were calculated in the malaria-infected and co-infected individuals and no differences in P. falciparum parasitaemia were observed between the malaria-infected and co-infected groups (Figure 5). Furthermore, no differences were observed in immune responses between the malaria-infected and co-infected groups for all parameters tested (Additional file 3; Figures 6 and 7).

\section{Discussion}

This study provides valuable insights into the immune responses associated with malaria infection in pre-school Nigerian children from 39-73 months. Studies looking at the immunological parameters in this age group are important given that the highest malaria mortality rates occur in children under five years [1]. Studies have shown that immune responses to malaria infection are established early in life and furthermore different ethnic groups respond differently to malaria infection and this is also established early in life [23]. There is little evidence of natural acquired immunity to malaria in children under two years as neonates less than 30 days old and children up to one year have reduced IFN- $\gamma$ producing capacity [1]. No increase in IFN- $\gamma$ levels was observed in the plasma of these children and this contradicts previous studies in children where increased levels of plasma IFN- $\gamma$ were observed [1]. In addition, a number of other factors associated with active malaria infection were not observed in these children.

Significantly high levels of IL-10 in plasma was observed from malaria-infected children and this corresponded with a reciprocal decrease in IL-12p70 levels, similar to that reported in other studies [24]. Regression analysis revealed a positive association between $P$. falciparum parasitaemia and IL-10. IL-10 is a known antagonist of this pro-inflammatory cytokine [25] and recurrent malaria infection can induce an immunosuppressive environment through secretion of high levels of IL-10, thus inhibiting Th1 responses and facilitating parasite persistence [26]. Intracellular IL-10 was not detected in polyclonally stimulated T-cell subsets from malaria-infected children when compared to endemic controls. PBMCs from malaria infected children when stimulated with $P$. falciparum parasites did no exhibit antigen specific immune responses. Previous studies have reported that $\mathrm{T}$-cell responses can be weak in young children infected with malaria and this lack of responses could explain why this age group is most susceptibility to infection [2].

The significant reduction in monocyte percentages in the infected groups compared to endemic controls was observed. Other reports have also shown a decrease in monocytes percentages during active parasitic infection [27]. During infection, circulating monocytes may be required at a higher rate to replenish resident macrophages and DCs. Alternatively, parasitic infections may induce monocyte apoptosis which could explain the 
observed decrease in monocyte percentages [28]. Despite the decreases in monocytes there was an increase in $\mathrm{CD}_{11 \mathrm{C}^{+}}$and $\mathrm{CD} 54^{+}$DCs in the infected individuals. The presence of predominantly mature $\mathrm{CD} 11 \mathrm{c}^{+} \mathrm{DCs}$ population may explain the lack of T-cell responses observed in young children as mature $\mathrm{CD} 11 \mathrm{c}^{+} \mathrm{DC}$ population lose its ability to phagocytose antigens which is necessary for presentation to effector and memory T-cells $[17,18]$. The increases in the percentage of $\mathrm{CD} 4^{+}$cells supports previous findings which demonstrated a link between increased CD54 expression and disease severity [28]. CD54 was previously shown to be upregulated on activated DCs, monocytes and other APCs during malaria infection in children and it is a known receptor that can bind $P$. falciparum erythrocyte membrane protein 1 [21]. CD11c and CD54 are expressed by many cell types and further analysis would shed light on the specific cell subset observed during infection.

Both $\gamma \delta$ T cells and NK cell express $\mathrm{CD}_{56}^{+}$and this surface maker increased in the malaria-infected children. NK cells and $\gamma \delta \mathrm{T}$ cells are thought to be critical in protection from malaria infection [29,30]. Depletion of these cells in murine malaria models has led to increased parasitaemia and delayed resolution of infection, emphasising their importance in early IFN- $\gamma$ production $[30,31]$. While these cells are more likely to act as accessory IFN- $\gamma$ secreting cells to effector $\mathrm{T}$ cells there was no increase in IFN- $\gamma$ detected in the plasma of infected children. However, recently, these cells have also been shown to act as APCs and compensate for DCs in certain situations and further studies would be required to determine the role of this subset $[31,32]$. While an increase in CD56 cell population was observed, further studies are required to determine the CD56 population subset and examine if these cells secrete IFN- $\gamma$.

While the study region is endemic for A. lumbricoides, no differences in immune parameters were observed between the co-infected groups compared to the $P$. falciparum-infected group only. Perhaps no differences were observed because children had a low intensity A. lumbricoides infection, as was demonstrated in a previous report study by Nacher et al. (2000). Moreover, since infection resides in the gut, perhaps only the medium and high intensity infection can alter the immune response systemically. Ascaris lumbricoides infection can be protective in the more severe forms of $P$. falciparum infection [33] and since individuals with severe malaria infection were excluded from the study an association could not be determined.

\section{Conclusion}

This data corroborates previous reports examining immune responses in malaria-infected children by showing that increases in IL-10 were positively associated with increased $P$. falciparum parasitaemia. Increases in innate immune cell populations that were previously associated with disease severity in malaria-infected individuals was also demonstrated $[19,21,33]$. Given that there are so few immunological studies in this age group, these findings could be useful in defining immune responses associated with increasing malaria parasitaemia in young children and therefore markers of disease susceptibility. It is estimated that $87 \%$ of children below the age of five are infected with malaria in the Osun State in south-western Nigeria [34], and validating these methodologies is important for future studies in this area. For example, birth to age five is an important range for the administration and study of many prophylactic paediatric vaccines and World Health Organization recommendations for routine immunization within this age group in Nigeria include both measles and yellow fever vaccines [35].

\section{Additional files}

Additional file 1: Sample of gating strategy for T Cells. Shown is a PBMC sample stimulated with $50 \mathrm{ng} / \mathrm{ml}$ PMA and $1 \mathrm{mg} / \mathrm{ml}$ ionomycin for $4 \mathrm{~h}$ in the presence of BFA $(10 \mathrm{mg} / \mathrm{ml})$. Cell subsets were identified as CD3 cells and then analysed for expression of cytokines and markers үocells.

Additional file 2: The average age, parasitemia, cytokine, nitric oxide (NO), RANTES, metalloproteinase (MMP) type 8 and tissue inhibitor of metalloproteinase (TIMP) type 1 plasma concentrations from the study cohort classified according in infection status (uninfected, Ascaris only, malaria only, Ascaris and malaria.

Additional file 3: The average age, cytokine, nitric oxide (NO), RANTES, metalloproteinase (MMP) type 8 and tissue inhibitor of metalloproteinase (TIMP) type 1 plasma concentrations from the study cohort classified according to $P$ falciparum parasitaemia.

\section{Competing interests}

GlaxoSmithKline sponsored the drug albendazole, which was used in larger clinical trial. The authors declare that they have no competing interests. The authors also declare that they have no financial competing interests.

\section{Authors' contributions}

CN carried out all immunological experiments and drafted the manuscript. MP is our biostatistician and performed the statistical analysis. DJD provided assistance in the immunological assays, AA provided assistance in the immunological assays, PK collected clinical samples and provided the parasitological data, SFM collected clinical samples, SOA participated in the design of the study, $\mathrm{CH}$ conceived the study, and participated in its design and coordination. SMO'N conceived the immunological aspects of study, participated in its design and edited the manuscript. All authors read and approved the final manuscript.

\section{Acknowledgements}

We would like to thank the children, mothers and Obas in the study communities for their co-operation throughout the study. We express our gratitude to the field workers in Nigeria for their help with data collection, and acknowledge Carol McManus (DCU) for technical support. This work was supported by the Health Research Board, Ireland.

\section{Author details}

${ }^{1}$ Parasite Immune Modulation Group, School of Nursing and Human Sciences, Faculty of Science and Health, Dublin City University, Glasnevin Dublin 9, Ireland. ${ }^{2}$ Department of Zoology, School of Natural Sciences, Trinity 
College Dublin, Dublin 2, Ireland. ${ }^{3}$ Obafemi Awolowo University, lle-lfe, Nigeria.

Received: 10 October 2012 Accepted: 12 December 2012 Published: 7 January 2013

\section{References}

1. Snow RW, Guerra CA, Noor AM, Myint HY, Hay SI: The global distribution of clinical episodes of Plasmodium falciparum malaria. Nature 2005, 434:214-217.

2. Doolan DL, Dobano C, Baird JK: Acquired immunity to malaria. Clin Microbiol Rev 2009, 22:13-36.

3. Phillips $\mathrm{S}$ : Effector mechanisms against asexual erythrocytic stages of Plasmodium. Immunol Lett 1994, 41:109-114.

4. Su Z, Stevenson MM: Central role of endogenous gamma interferon in protective immunity against blood-stage Plasmodium chabaudi as infection. Infect Immun 2000, 68:4399-4406.

5. Day NP, Hien TT, Schollaardt T: The prognostic and pathophysiologic role of pro- and anti-inflammatory cytokines in severe malaria. $J$ Infect Dis 1999, 180:1288-1297.

6. Dodoo D, Omer FM, Todd J, Akanmori BD, Koram KA, Riley EM: Absolute levels and ratios of pro-inflammatory and anti-inflammatory cytokine production in vitro predict clinical immunity to Plasmodium falciparum malaria. J Infect Dis 2002, 185:971-979.

7. John CC, Opika-Opoka R, Byarugaba J, Idro R, Boivin MJ: Low levels of RANTES are associated with mortality in children with cerebral malaria. $J$ Infect Dis 2006, 194:837-845.

8. Were T, Hittner JB, Ouma C, Otieno RO, Orago AS, Ong'echa JM, Vulule JM, Keller CC, Perkins DJ: Suppression of RANTES in children with Plasmodium falciparum malaria. Haematologica 2006, 91:1396-1399.

9. Dietmann A, Helbok R, Lackner P, Issifou S, Lell B, Matsiegui PB, Reindl M, Schmutzhard E, Kremsner PG: Matrix metalloproteinases and their tissue inhibitors (TIMPs) in Plasmodium falciparum malaria: serum levels of TIMP-1 are associated with disease severity. J Infect Dis 2008, 197:1614-1620.

10. Kirwan P, Jackson AL, Asaolu SO, Molloy SF, Abiona TC, Bruce MC, RanfordCartwright L, O' Neill SM, Holland CV: Impact of repeated four-monthly anthelmintic treatment on Plasmodium infection in preschool children: a double-blind placebo-controlled randomized trial. BMC Infect Dis 2010, 10:277.

11. Kirwan P, Asaolu SO, Molloy SF, Abiona TC, Jackson AL, Holland CV: Patterns of soil-transmitted helminth infection and impact of four-monthly albendazole treatments in preschool children from semi-urban communities in Nigeria; a double-blind placebo-controlled randomised trial. BMC Infect Dis 2009, 9:20.

12. Lyke KE, Dabo A, Sangare L: Effects of concomitant Schistosoma haematobium infection on the serum cytokine levels elicited by acute Plasmodium falciparum malaria infection in Malian children. Infect Immun 2006, 74:5718-5724.

13. Nacher M, Gay F, Singhasivanon P: Ascaris lumbricoides infection is associated with protection from cerebral malaria. Parasite Immunol 2000 22:107-113.

14. De Silva NR, Brooker S, Hotez PJ, Montresor A, Engels D, Savioli L: Soiltransmitted helminth infections: updating the global picture. Trends Parasitol 2003, 19:547-551.

15. Elliott SR, Spurck TP, Dodin JM: Inhibition of dendritic cell maturation by malaria is dose dependent and does not require Plasmodium falciparum erythrocyte membrane protein 1. Infect Immun 2007, 75:3621-3632.

16. Randolph GJ, Inaba K, Robbiani DF, Steinman RM, Muller WA: Differentiation of phagocytic monocytes into lymph node dendritic cells in vivo. Immunity 1999, 11:753-761.

17. Banchereau J, Briere F, Caux C: Immunobiology of dendritic cells. Annu Rev Immunol 2000, 18:767-811.

18. Leisewitz AL, Rockett KA, Gumede B, Jones M, Urban B, Kwiatkowski DP: Response of the splenic dendritic cell population to malaria infection. Infect Immun 2004, 72:4233-4239.

19. Sponaas AM, Cadman ET, Voisine C, Harrison V, Boonstra A, O'Garra A Langhorne J: Malaria infection changes the ability of splenic dendritic cell populations to stimulate antigen-specific T cells. J Exp Med 2006, 203:1427-1433.
20. Jenkins NE, Chakravorty SJ, Urban BC, Kai OK, Marsh K, Craig AG: The effect of Plasmodium falciparum infection on expression of monocyte surface molecules. Trans R Soc Trop Med Hyg 2006, 11:1007-1012.

21. D'Ombrain MC, Hansen DS, Simpson KM, Schofield L: gammadelta-T cells expressing NK receptors predominate over NK cells and conventional T cells in the innate IFN-gamma response to Plasmodium falciparum malaria. Eur J Immunol 2007, 37:1864-1873.

22. Boström S, Giusti P, Arama C, Persson JO, Dara V, Traore B, Dolo A, Doumbo O, Troye-Blomberg M: Changes in the levels of cytokines, chemokines and malaria-specific antibodies in response to Plasmodium falciparum infection in children living in sympatry in Mali. Malar J 2012, 11:109.

23. Prakash D, Fesel C, Jain R, Cazenave PA, Mishra GC, Pied S: Clusters of cytokines determine malaria severity in Plasmodium falciparum-infected patients from endemic areas of Central India. J Infect Dis 2006, 194:198-207.

24. Trinchieri G: Interleukin-12 and the regulation of innate resistance and adaptive immunity. Nat Rev Immunol 2003, 3:133-146.

25. Cooper PJ, Moncayo AL, Guadalupe I: Repeated treatments with albendazole enhance Th2 responses to Ascaris Lumbricoides, but not to aeroallergens, in children from rural communities in the Tropics. I Infect Dis 2008, 198:1237-1242

26. Semnani RT, Venugopal PG, Mahapatra L, Skinner JA, Meylan F, Chien D, Dorward DW, Chaussabel D, Siegel RM, Nutman TB: Induction of TRAILand TNF-alpha-dependent apoptosis in human monocyte-derived dendritic cells by microfilariae of Brugia malayi. J Immunol 2008 181:7081-7089.

27. Loharungsikul S, Troye-Blomberg M, Amoudruz P, Pichyangkul S, Yongvanitchit K, Looareesuwan S, Mahakunkijcharoen Y, Sarntivijai S, Khusmith S: Expression of toll-like receptors on antigen-presenting cells in patients with falciparum malaria. Acta Trop 2008, 105:10-15.

28. Cserti-Gazdewich CM, Dzik WH, Erdman L, Ssewanyana I, Dhabangim A, Musoke C, Kain KC: Combined measurement of soluble and cellular ICAM-1 among children with Plasmodium falciparum malaria in Uganda. Malar J 2010, 9:233.

29. Choudhury HR, Sheikh NA, Bancroft GJ, Katz DR, De Souza JB: Early nonspecific immune responses and immunity to blood-stage nonlethal Plasmodium yoelii malaria. Infect Immun 2000, 68:6127-6132

30. Mohan K, Moulin P, Stevenson MM: Natural killer cell cytokine production, not cytotoxicity, contributes to resistance against blood-stage Plasmodium chabaudi AS infection. J Immunol 1997, 159:4990-4998.

31. Hanna J, Gonen-Gross T, Fitchett J: Novel APC-like properties of human NK cells directly regulate T cell activation. J Clin Invest 2004, 114:1612-1623.

32. Moser B, Brandes M: Gammadelta T cells: an alternative type of professional APC. Trends Immunol 2006, 27:112-118.

33. Nacher N: Interactions between worms and malaria: Good worms or bad worms? Malar J 2011, 10:259

34. Olasehinde Gl, Ajayi AA, Taiwo SO, Adekeye BT, Adeyeba OA: Prevalence and management of falciparium malaria among infants and children in Ota, Ogun State, Southwestern Nigeria. African Journal of Clinical and Experimental Microbiology 2010, 11:159-163.

35. Sanchez-Schmitz G: Development of newborn and infant vaccines. Sci Transl Med 2011, 3(90ps):27.

doi:10.1186/1475-2875-12-5

Cite this article as: Noone et al:: Plasma cytokines, chemokines and cellular immune responses in pre-school Nigerian children infected with Plasmodium falciparum. Malaria Journal 2013 12:5. 